\title{
Correction to: Epigenetic Basis of Circadian Rhythm Disruption in Cancer
}

\section{Edyta Reszka and Shanbeh Zienolddiny}

\section{Correction to:}

Chapter 10 in: Ramona G. Dumitrescu and Mukesh Verma (eds.), Cancer Epigenetics for Precision Medicine: Methods and Protocols, Methods in Molecular Biology, vol. 1856, https://doi.org/10.1007/978-1-4939-8751-1_10

The Chapter was inadvertently published with an incorrect version of (Fig. 1). The figure has been corrected in the chapter.

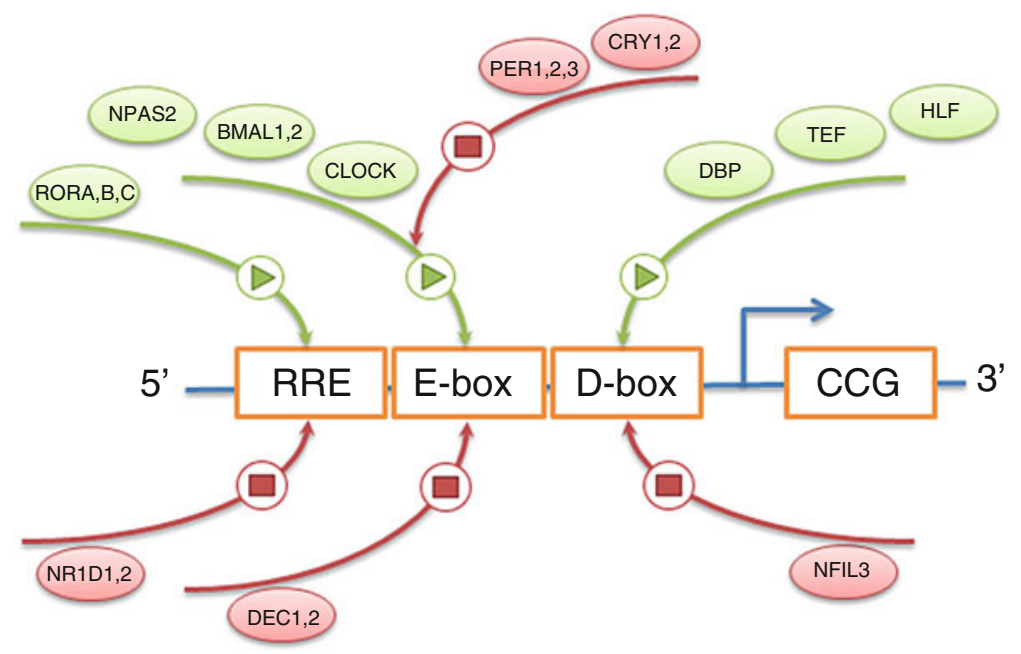

Fig. 1 Circadian promoter elements: E-box, D-box, ROR-element (RRE) and their activators and repressors in clock-controlled gene (CCG) transcription 